\title{
Acute Resistance Exercise Program Variables and Subsequent Hormonal Response
}

\author{
Lawrence D Hayes ${ }^{1,2}$, Gordon F Bickerstaff ${ }^{3}$ and Julien S Baker ${ }^{1 *}$ \\ ${ }^{1}$ Institute of Clinical Exercise and Health Science, University of the West of Scotland, Scotland \\ ${ }^{2}$ School of Human Sciences, London Metropolitan University, Scotland \\ ${ }^{3}$ Department of Biology, University of the West of Scotland, Scotland
}

\begin{abstract}
Resistance Exercise (RE) is a widely practiced activity both in leisure time and in training periods for competitive athletes. Recent advanced in molecular biology and muscle physiology has elucidated some of the mechanisms that regulate muscle growth. However, these molecular advances require application in acute program variables of RE. Therefore, we present an updated paradigm of resistance exercise variables and the effect manipulating these has on signalling pathways and hormonal response to optimise RE adaptations. We herein explore the effect of altering (i) choice of exercise; (ii) order of exercises performed; (iii) load (weight/resistance); (iv) volume (i.e. repetitions $x$ sets $\mathrm{x}$ load) and (v) rest period between sets. Manipulating these variables has a consequential effect on signalling pathways, hormone response and neural adaptations that may influence protein synthesis and therefore gradual protein accretion leading to increased muscle size and strength.
\end{abstract}

Keywords: Resistance exercise; Weight training; Growth hormone; Testosterone; Cortisol

\section{Introduction}

Resistance Exercise (RE) is one of the most widely practiced forms of physical activity. This type of exercise is used to enhance athletic performance, augment musculo-skeletal health and alter body aesthetics. The health benefits of RE are primarily as a preventative or countermeasure to circumstances where muscle weakness compromises optimal function (injury or prolonged inactivity, sarcopenia or musculoskeletal disorders) however, it also has a positive effect on skeletal and metabolic health as well as potential psychological benefits. This review addresses factors that can impact upon RE outcomes and attempts to outline recommendations for favourable physiological adaptations.

\section{Training Design}

Acute RE program variables, originally defined by Kraemer characterise the type and magnitude of the RE stimulus [1]. These variables are: (i) choice of exercise; (ii) order of exercises performed; (iii) load (weight/resistance); (iv) volume (i.e. repetitions $\mathrm{x}$ sets $\mathrm{x}$ load) and (v) rest period between sets.

Choice of exercise: Exercise selection determines the muscle group exercised and the speed of muscle contraction (eccentric, concentric and/or isometric). Multiple-joint exercises such as bench press and squat require complex neural responses and have generally been found most effective for overall strength increases because they allow greater weight to be lifted [2,3]. Exercising large muscle groups provides a larger hormone response than exercising small muscle groups $[4,5]$. An increased level of hypertrophy in response to RE has previously been reported in the upper body muscles compared to lower extremity muscles [6]. Welle et al. [6] reported Anatomical Cross-Sectional Area (ACSA) of elbow flexors to increase by $9 \%$ and $22 \%$ in old and young subjects, respectively; whereas, knee extension ACSA increased by only $6 \%$ and $4 \%$ respectively. Abe et al. [7] found a similar response when assessing muscle thickness by ultrasound. A logical explanation for this is that the lower body musculature is habitually activated and loaded to a higher level during diurnal activities than the upper body musculature [8]. Alternatively, Kadi et al. [9] suggested that intramuscular differences in androgen receptor concentrations may explain the increased potential for upper body hypertrophy.

Muscle contraction velocity is inversely related to the capacity for maximum load [10]. However, isokinetic training has been shown to increase muscular strength specific to the training velocity [11] yet, training at a moderate velocity $\left(180-240^{\circ} \cdot \mathrm{s}^{-1}\right)$ produces the greatest carryover of strength [12]. The velocity of muscular contraction also impacts signalling responses and adaptations to RE [13]. Eccentric contractions noticeably increase the $70 \mathrm{kDa}$ ribosomal protein $\mathrm{S} 6$ kinase (p70 S6K) phosphorylation, while maximal concentric and isometric contractions provide smaller effects [14]. Additionally, eccentric muscle actions stimulate mitogen-activated protein kinase (MAPK) signalling [15]. When force is equated, concentric, isometric and eccentric actions produce similar signalling responses [16], however it is well reported that skeletal muscle can generate $~ 30 \%$ more tension during eccentric contractions than during concentric muscle actions [14]. This inequality in muscle tension has been proposed as an explanation for greater signalling response during eccentric contractions [14]. Furthermore, hypertrophy is attenuated after concentric only RE (matched with conventional concentric/eccentric for total work) [17]. Together, these studies suggest that signalling is similar for all muscle contraction velocities. However, force production capacity is greater during eccentric contractions and may be a more potent stimulus for muscle signalling. Although inclusion of eccentric contractions may be beneficial for RE adaptations, excessive eccentric muscle actions may produce excessive muscle damage. However, this myofibrillar disruption may be causally related to hypertrophy but this is still speculative.

Order of Exercises Performed: Exercise order significantly affects

*Corresponding author: Julien S Baker, Professor, Director, Institute of Clinical Exercise and Health Science, Chair \& Head of Sport and Exercise Science Subject Development Group, Exercise Science Research Laboratory, School of Science, Faculty of Science and Technology, University of the West of Scotland, Hamilton Lanarkshire, ML3 OJB, Scotland, Tel: 01698 283100; Fax: 01698 894404; E-mail: jsbaker@uws.ac.uk

Received August 12, 2013; Accepted August 23, 2013; Published August 30, 2013

Citation: Hayes LD, Bickerstaff GF, Baker JS (2013) Acute Resistance Exercise Program Variables and Subsequent Hormonal Response. J Sports Med Doping Stud 3: 125. doi:10.4172/2161-0673.1000125

Copyright: $\odot 2013$ Hayes LD, et al. This is an open-access article distributed under the terms of the Creative Commons Attribution License, which permits unrestricted use, distribution, and reproduction in any medium, provided the original author and source are credited. 
the acute expression of muscular strength [18]. Spreuwenberg et al. [18] reported that performing a squat last, as opposed to first during a RE session significantly reduced the number of repetitions performed. Strength and power during multiple joint exercises may be reduced when performed after several other exercises in a RE session [19]. A reduction in muscle activation (measured by electromyography) and metabolic fatigue (reductions in glycogen and/or phosphocreatine) are likely explanations for reduced strength and/or muscular endurance $[13,20]$. Interestingly, when exercises are sequenced based on agonist/ antagonist muscle group relationships, muscle force and power may be potentiated [21]. Considering that multiple-joint exercises have been shown effective for increasing strength and hypertrophy, maximising performance of these exercises early in a workout may be necessary for optimal strength gains [18]. In fact, the American College of Sports Medicine Position Stand recommends that workouts include large muscle groups before small muscle groups, multiple-joint before singlejoint exercises, high-intensity before lower-intensity exercises [22]. The same authors offer alternative recommendations; rotation of upper and lower body exercises or agonist-antagonist exercises (commonly known as "supersets").

Load: Load represents the amount of weight lifted or the resistance used during an exercise. Altering this load affects the acute metabolic hormonal neural and cardiovascular responses to RE [23-26]. The maximal load that can be used is heavily dependent on other acute program variables such as exercise order, exercise selection (specifically the muscle action), volume and rest interval length. Load is typically prescribed as a percentage of one repetition maximum (1RM) (e.g. $80 \% 1 \mathrm{RM})$ or as a weight that allows a certain number of repetitions (e.g. 6RM). Typically in RE, there is an inverse relationship between the load and the volume (i.e. as the load increases the number of repetitions decreases). Loads of below 50\% 1RM have been shown to increase dynamic muscular strength in untrained individuals $[27,28]$. Hakkinen et al. [26] reported that at least $80 \% 1 \mathrm{RM}$ was required to stimulate further neural adaptations and strength during RE in experienced weight lifters. In agreement with these findings, several authors indicated that loads of approximately 5-6RM were optimal for increasing maximal dynamic strength [29-31]. Although significant increases in strength have been reported using loads of 8-12RM [32,33], these loads may be insufficient to stimulate adaptations in experienced weight lifters $[26,32,33]$.

In RE research, rarely is the effect of load on muscular adaptations examined without being affected by confounding variables such as volume. However, Low-Frequency Electrical Stimulation (LFES, simulating low-force contractions) and High-Frequency Electrical Stimulation (HFES, simulating high-force contractions) allow the effect of load on RE adaptations to be analysed [34,35]. LFES increases Adenosine Monophosphate-Activated Protein Kinase (AMPK) activity which promotes glucose and fatty acid oxidisation and inhibits protein synthesis (Figure 1). Simply, AMPK promotes energy release and inhibits energy consumption when cellular energy is low (marked by high Adenosine Monophosphate (AMP) and low glycogen) [13]. HFES stimulates protein kinase B (Akt)-mammalian target of rapamycin (mTOR) signalling. This response is critical for increasing muscle protein synthesis and subsequent hypertrophy $[36,37]$. mTOR signalling increases protein synthesis by increasing the number of messenger RNA translated per ribosome [38]. According to Henneman's size principal smaller motor units (primarily type I) are recruited prior to larger motor units (primarily type II) until force production is equal to force requirement [39]. Low-force activities recruit primarily type I, slow fatiguing motor units. As the load increases, higher

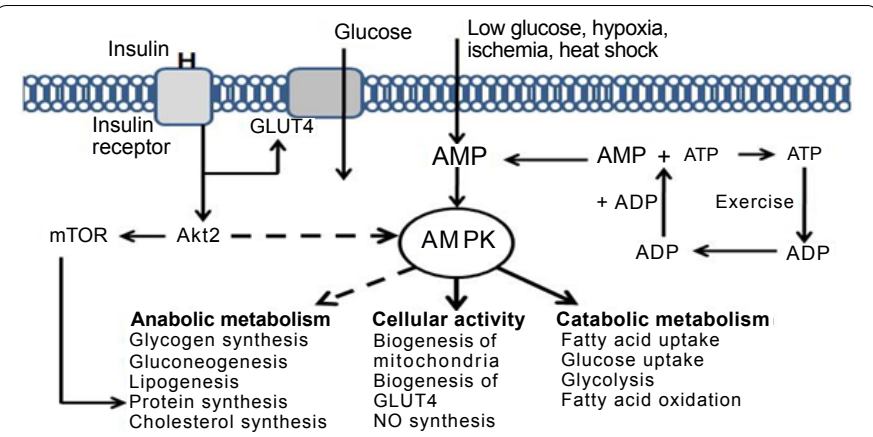

Figure 1: AMPK activity and subsequent metabolic effects.

threshold motor units are recruited, encompassing more type II fastfatiguing fibres until near maximal-loads where the entire spectrum of motor units is activated. As the size principal is related to force, and force $=$ mass $\times$ acceleration, rapidly accelerating a load can recruit higher-threshold motor units [40]. Linnamo et al. [41] reported that performing 'explosive' concentric contractions using $~ 40 \%$ of maximal isometric force increased Electromyographic (EMG) activity when compared with the same exercise at $\sim 67 \%$ of maximal isometric force at a slow velocity. EMG activity has also been found to increase with the onset of fatigue [42]. This indicates increased contribution of higher threshold motor units to maintain force output as lower threshold units begin to fatigue. Therefore, a heavy weight, 'explosive' contractions, or fatigue may all increase the number of type II motor units recruited. This may affect the adaptations to RE as only motor units recruited respond to RE type II fibres have a greater capacity for hypertrophy and different muscle groups contain varied percentages of type I and type II fibres [43]. RE to maximize hypertrophy typically include moderate to high loads $[13,43-45]$. These loads allow the volume to be high and the rest periods to be short to maximize hormonal response (Figure 1) [44].

Traditionally, 1-6RM, 8-12RM and 15+RM loads are recommended to maximize strength, hypertrophy and local muscular endurance, respectively. Campos et al. confirmed these preconceptions by investigating the adaptations following an 8 week RE program in groups using 3-5RM loads (strength protocol), 9-11RM loads (hypertrophy protocol) and 20-28RM loads (muscular endurance protocol) [28] The investigation revealed a step-wise increase in strength (strength protocol $>$ hypertrophy protocol $>$ muscular endurance protocol), local muscular endurance (muscular endurance protocol>hypertrophy protocol $>$ strength protocol). Increased muscle fiber Cross-Sectional Area (CSA) occurred only in the hypertrophy and strength protocol groups with no significant difference observed between the two. Maximal aerobic power increased solely in the muscular endurance protocol group. Campos et al. however, did not control for volume of training, which has been suggested as a strong indicator for muscular adaptations $[28,46]$

Research examining periodization has demonstrated a need for variable-intensity loading schemes in RE [45,47]. Rhea et al. reported that daily undulating periodization elicited greater strength gains than the classic linear schedule [48]. An example of daily undulating periodization would be training at 70,85 , and $95 \%$ of $1 \mathrm{RM}$ on Monday, Wednesday, and Friday, respectively. The mechanisms behind increased strength due solely to periodization are unclear. Selye's General Adaptation Syndrome suggests that a system will adapt to an unaccustomed stress (i.e. overload). Greater volumes and intensities of $\mathrm{RE}$ result in strength adaptations to a certain level presumably, due to an overload of the neuromuscular system $[46,49]$. Therefore, variations 
in training may increase the neuromuscular overload by continually applying an unaccustomed stress. The American College of Sports Medicine Position Stand recommends that workouts cycle training loads of $80-100 \% 1 \mathrm{RM}$ to maximize muscular strength and $70-100 \%$ $1 \mathrm{RM}$ to maximize hypertrophy [22].

Volume: Exercise volume is typically described as the total number of repetitions performed during a training session multiplied by the resistance used and is reflective of the duration which muscles are being stressed [50]. Therefore, manipulation of training volume can be achieved by altering the number of exercises per session, the number of sets per exercise, the number of repetitions per set, or the resistance used. Volume has been shown to affect neural hypertrophic metabolic and hormonal responses to RE [22,24,50,51]. For example, multipleset workouts invoke significantly greater Growth Hormone (GH) and Testosterone (T) responses than single-set programs [52]. Long-term training that employs multiple sets, compared to single sets appears to be superior for strength development and hypertrophy [51]. It is unclear however, whether a dose response exists between volume and RE adaptations. Studies incorporating two three four to five and six or more sets per exercise have all produced significant increases in strength in trained and untrained individuals. Interestingly, similar strength increases have been reported when comparing two and four sets and two and three sets [32,33,53-57]. In contrast to Ostrowski et al. [56], Berger [29] reported that three sets were indeed superior to two sets for the development of strength. The optimal number of sets per exercise still remains an equivocal matter with varied opinions even between review and meta-analytical papers. A recent meta-regression reported that multiple sets were associated with a larger effect size than a single set [58]. In a dose-response manner, two to three sets per exercise were associated with a significantly greater effect size than one set. However, no significant difference between one set per exercise and four to six sets per exercise, or between two to three sets per exercise and four to six sets per exercise was observed. Despite no significant difference observed between one set and four to six sets, a trend for four to six sets to result in greater strength increases existed. This was not significant due to the large standard deviation observed. The authors concluded by advising that two to three sets per exercise are associated with $46 \%$ greater strength gains than one set, in both trained and untrained subjects. An alternative view has been proposed by Peterson et al. [46], who reported that approximately eight sets per muscle group produced the largest effect size in athletes. Peterson et al. [46] investigated 177 studies and 1,803 effect sizes and advised the following RE prescription: For athletes, maximal strength gains are elicited at a mean training intensity of $80 \%$ of $1 \mathrm{RM}$ (agreeing with the American College of Sports Medicine Position Stand) two days per week, with a training volume of eight sets per muscle group [22]. RE trained nonathletes exhibit maximal strength gains with a training intensity of $80 \% 1 \mathrm{RM}, 3$ days per week, with a training volume of 4 sets per muscle group. Untrained individuals experienced maximal strength gains at $60 \% 1 \mathrm{RM}$, three days per week and four sets per muscle group. These authors demonstrated that exercise prescription for maximum strength gains should vary between populations. However, trained non athletes commonly train for hypertrophy rather than strength and therefore, the above prescriptions may not be relevant to this population.

Hypertrophy involves a proportionate increase in the net accretion of actin and myosin as well as other structural proteins. Mechanical loading leads to a series of intracellular events that ultimately regulate gene expression and protein synthesis [22]. Protein synthesis in human muscle increases after only one bout of RE and peaks approximately 24 $h$ post exercise [59]. This anabolic environment remains elevated from $\sim 2 \mathrm{~h}$ post exercise up through 36-48 h post exercise [60] (depending on training status). For previously untrained individuals neural adaptations dominate early stages of training with muscle hypertrophy occurring within six weeks [61]. Similarly to strength training, hypertrophy has shown to be enhanced with multiple-set over single-set training [23]. In untrained individuals however, as with strength, general nonspecific program design appears to be an adequate overload to stimulate hypertrophy [62]. For more experienced individuals, periodized training schemes appear to be the most effective way to maximize hypertrophy. RE targeting hypertrophy commonly include moderate to very high loading, relatively high volume, and short rest intervals as this design produces a greater elevation in $\mathrm{T}$ and $\mathrm{GH}$ than a strength program with long rest, high-load and low-volume [22]. Total work, in combination with loading has been implicated for gains in hypertrophy [63]. This result has been supported by greater hypertrophy associated with multiple-set, high-volume programs compared to single-set programs $[33,64]$. Goto et al. reported that the addition of a highrepetition low-load set to a traditional strength training protocol increased muscle CSA [65]. However, this combination protocol was compared with a strength only protocol and a typical hypertrophy regimen was not analysed. Campos et al. examined eight weeks of a high-load RE protocol and reported that neither type I nor type II muscle fibres exhibited hypertrophy [28]. Therefore, the American College of Sports Medicine Position Stand advised that a combination of strength training and typical hypertrophy training is most effective for advanced hypertrophy training as it compromises a load sufficient to overload the musculature and a volume high enough to elicit an elevated endocrine response [22]. As with many RE variables, volume is difficult to isolate as and therefore most experiments manipulate load and volume concomitantly. It seems logical however, that increased load would affect muscle signalling. High-volume RE would deplete muscle glycogen stores and therefore stimulate AMPK activity [13]. As previously reported AMPK promotes muscular endurance adaptations and inhibits protein synthesis (via inhibition of Akt-mTOR signalling). This relationship between volume, glycogen, and AMPK is supported by research that demonstrates that low muscle glycogen potentiates exercise-induced AMPK activity and attenuates Akt signalling [64-66]. Comparatively, adequate exercise volume appears necessary for optimal gains in muscular strength and size [51]. Therefore, making conclusions and recommendations about RE volume and muscle signalling is difficult and a further research to establish the threshold RE volume at which AMPK begins to inhibit Akt-mTOR signalling is required.

Rest intervals: Rest periods between sets significantly influence the adaptations and responses to RE. Short rest periods are recommended for hypertrophy as short rest periods augment the GH response [67]. However, short rest intervals have a detrimental effect of subsequent sets and over several weeks, attenuate strength increases $[42,68]$. When training to optimise strength gains, longer rest periods are advised as performance may be compromised with one-min compared to three min rest periods [69]. Strength recovery may not even be complete after 2 mins with several studies suggesting that $3-5$ min rest interval produce less performance decrements than $30 \mathrm{~s}-2 \mathrm{~min}[23,71,70]$. The American College of Sports Medicine Position Stand advised rest periods of at least $2-3 \mathrm{~min}$ for core exercises using heavy loads (e.g. Olympic lifts) and one - two min for assistance exercises for the development of strength [22]. The authors advised identical rest periods to maximize hypertrophy in advanced RE training. The aim of hypertrophy training is to produce an anabolic environment, which appears to be best done with short rest periods. However, Robinson et al. [72] reported no difference in muscle girths, body mass or skin folds in recreationally trained participants when utilising 30,90 and $180 \mathrm{~s}$ 
rest intervals. In agreement, Ahtiainen et al. [73] found no difference in muscle CSA after three months of training with 5 min or 2 min rest periods. Intuitively, short rest periods would increase AMPK activity due to the metabolic stress associated with little rest and therefore inhibit protein synthesis following RE, explicating why short rest periods attenuate strength gains $[42,69]$. However, there is some debate as to whether short rest periods inhibit protein synthesis via AMPK activity as GH is increased with short rest periods, therefore promoting hypertrophy. Also, the importance of increased AMPK activity $\sim 1-2$ $\mathrm{h}$ post-exercise remains unclear [13]. Regardless of these indefinites, short rest periods are commonly prescribed when hypertrophy is the aim $[13,22]$.

\section{Resistance training and the endocrine system}

Dependent upon RE variables, RE incites an anabolic hormonal response, including T, GH and Insulin-Like Growth Factor-1 (IGF-1), but also Cortisol (C; a catabolic hormone) [22]. The acute program variables discussed in section 1 dictate the magnitude of this hormonal response. Typical hypertrophy protocols (moderate to high load, short rest periods and high volume) recruiting large muscle groups appear to produce the greatest endocrine response (Figure 2) [3].

\section{Testosterone}

$\mathrm{T}$ is considered to be of high importance in resistance training adaptations in men [74]. $\mathrm{T}$ is a steroid hormone secreted from the Leydig cells of the testes under hypothalamic and pituitary control. T has both anabolic and anticatabolic effects on muscle tissue [75]. The direct action of $\mathrm{T}$ on muscle is supported by the presence of cytoplasmic receptors for $\mathrm{T}$ in skeletal muscle homogenates [76]. T promotes protein synthesis in muscle tissue with the result being increased muscle mass and strength [77]. Kvorning et al. [78] suppressed endogenous production of $\mathrm{T}$ with goserelin in healthy young males and reported serum $\mathrm{T}$ below $10 \%$ of normal levels strongly attenuated the increase in lean mass and muscle strength and actually increased fat mass during a resistance training protocol. The placebo group in the study adapted to the resistance training period by significantly increasing isometric strength and lean leg mass. These results demonstrate a direct link between endogenous $\mathrm{T}$ and adaptations to resistance training. Hansen et al. [79] subjected 16 untrained men to nine weeks of training either unilateral arm alone, or unilateral arm and legs. It was reported that the group who training both legs and arm in the same training session exhibited a mean isometric elbow flexion strength gain of $37 \%$ compared to $10 \%$ in the arm only group after nine weeks. In fact, even the untrained arm showed a significant increase in strength for the legs and arm group over the nine week training program. In contrast, West et al.

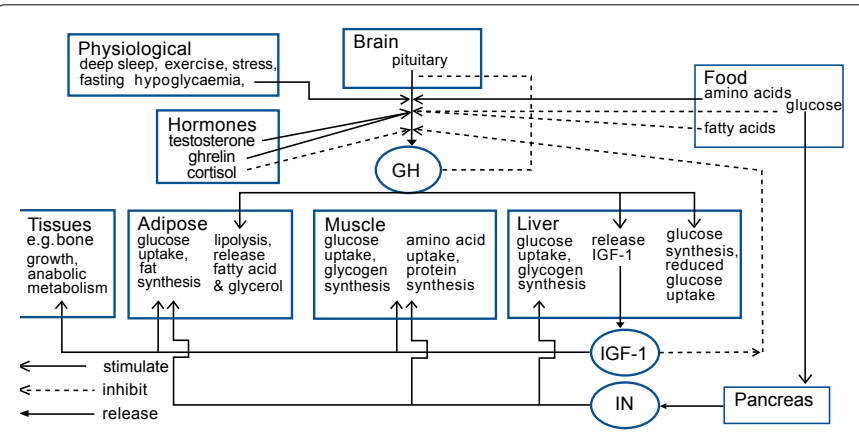

Figure 2: Schematic map of the various hormone, physiological and foodderived factors that influence release of Growth Hormone $(\mathrm{GH})$, and the main effects of GH, Insulin-like Growth Factor-1 (IGF-1), Insulin (IN) and GH on some of the key the metabolic processes that occur in various tissue cells.
[5] conducted a similar study but utilised a within-participants design and noted that despite a higher hormonal response to training, subjects achieved significantly indifferent hypertrophy and strength gains when training one arm and legs compared to one arm alone. These authors summarised by suggesting that acute systemic hormone responses are not responsible for muscle hypertrophy and that local mechanisms are primary in producing gains in strength and hypertrophy. However, West et al. [5] tested strength dynamically, whereas Hansen et al. [79] tested isometric strength and this may explain some difference.

The correlation reported by Ahtiainen et al. [80] between changes in isometric strength and T suggest that $\mathrm{T}$ may be an important factor for strength development. Interestingly, participants who exhibited increased acute $\mathrm{T}$ response after the training period were able to increase CSA of muscle more than those with a lowered response. However, caution should be exerted when examining $\mathrm{T}$ concentrations correlated to strength or hypertrophy as $\mathrm{T}$ response to resistance exercise is transient and the accretion of protein leading to hypertrophy and strength increases are accumulative. Although $\mathrm{T}$ causes up regulation of androgen receptors, the increase in $\mathrm{T}$ is transitory and muscle protein synthetic response can be elevated for $\sim 48 \mathrm{~h}$ although this time course is reduced with training $[13,81]$. The hormone response to resistance training has been repeatedly investigated with the current theory of interaction between endogenous $\mathrm{T}$ and androgen receptors in the recovery phase stimulating muscle hypertrophy, protein synthesis and strength $[4,74,80]$. This is furthermore supported by the fact that exogenous $\mathrm{T}$ (via supplementation) significantly increases muscle mass and strength, especially when combined with resistance training [82] A reduction in $\mathrm{T}$ may cause an increased storage of fat via decreased fat oxidation, decreased resting energy expenditure, and increased adiposity $[83,84]$.

Despite many authors suggesting that resistance exercise-induced hypertrophy is attributable to acute hormonal response recent findings have suggested otherwise $[4,25,79,85,86]$. West et al. [86] found that myofibrillar protein synthesis and phosphorylation of the $70 \mathrm{kDa} S 6$ protein kinase (both thought to be predictors of hypertrophy) exhibited no difference between arm only and arm and legs training. The latter protocol elicited a marked elevation in serum T, GH and insulin-like growth factor-1 (IGF-1) from resting values, whereas the former did not. However, participants were supplemented with $25 \mathrm{~g}$ whey protein after both protocols which led West et al. [5] to suggest that high quality protein availability, rather than training designed to elevate hormone levels is crucial for muscle hypertrophy.

$\mathrm{T}$ indirectly stimulates secretion of IGF-1 and GH, known to be correlated with the magnitude of type I and type II muscle fiber hypertrophy [87]. Numerous studies have demonstrated that resistance exercise increase circulating $\mathrm{T}$ concentrations $[5,9,25,85]$. T exerts its influence on protein synthesis via androgen receptors in the muscle. $\mathrm{T}$ binds to and converts androgen receptors to a sequence-specific DNA binding factor capable of transferring to the nucleus and associating with DNA to regulate androgen-specific gene expression. Blocking of androgen receptors reduces muscle protein accretion, indicating the physiological importance of $\mathrm{T}$-androgen receptor interactions for hypertrophy [88]. Androgen receptors are up regulated by $\mathrm{T}$ therefore the higher $\mathrm{T}$ concentrations in men likely explains the higher androgen receptor content found in men compared to women $[38,89]$. Kraemer et al. [90] reported that a resistance training protocol significantly increased resting testosterone concentrations and that this was augmented by essential amino acid supplementation. This would suggest that androgen receptors were up regulated as a result of this and indeed, hypertrophy (as a result of protein accretion) did occur 
as a result of the 12-week training protocol. While endurance exercise may transiently increase $T$ values the number of muscle fibres recruited is less than during resistance training and androgen receptors are only impacted if the motor unit is activated [91]. Therefore, endurance training is less effective at promoting hypertrophy than resistance exercise as resistance exercise (particularly when working to failure) recruits more muscle fibres [13].

It has previously been reported that $\mathrm{T}$ responses to training may affect individual adaptation and the ability to exhibit hypertrophy $[25,78,85]$. For example, Kraemer et al. [92] reported that protocolspecific acute serum $\mathrm{T}$ increases were observed after strength and hypertrophy protocols. However, the direction and amplitude of hormonal responses has been shown to vary even in response to similar resistance training protocols $[25,68,85]$. Beaven et al. [25] investigated the effect of four different resistance exercise protocols on hormonal response in elite rugby players and reported large inter-individual variability for serum T. None of the four protocols: strength ( $3 \mathrm{X} 5$ reps at $85 \% 1 \mathrm{RM}$ ), hypertrophy ( $4 \mathrm{X} 10$ reps at $70 \% 1 \mathrm{RM})$, power ( $3 \mathrm{X} 5$ reps at $40 \% 1 \mathrm{RM}$ ) and strength endurance (5 X 15 reps at $55 \% 1 \mathrm{RM})$ induced a statistically significant increase in $\mathrm{T}$. This was probably due to the large inter-individual variability within the study. The strength endurance and power protocols in the Beaven et al. [25] study elicited lower $\mathrm{T}$ responses than the strength and hypertrophy protocols. However, significance was not reached as there was large variability in hormonal responses to resistance exercise as indicated by large standard errors. The authors noted, however, that individual's hormonal response was pronounced in one or occasionally two of the protocols implemented. Therefore, the protocol considered to be optimal in terms on anabolism (defined by an absolute increase in free T concentration) varied amongst individuals. These investigators were the first to observe this phenomenon and offered it as a potential reason why studies have varied in terms of observed hormonal response to resistance exercise. Beaven et al. [25] reported four groups of responders (hypertrophy: $n$ =4; strength: $n=5$; strength endurance: $n=4$; power: $n=2$ ). If these findings were representative across a wider population, pooling data would likely elicited skewed results depending on subject homogeneity.

As suggested by Beaven et al. [25] different resistance exercise protocols may result in individualised $\mathrm{T}$ responses and as already suggested, a relationship between $\mathrm{T}$ response and muscular adaptations has been suggested [78]. Therefore, Beaven et al. [85] assigned a group of amateur rugby players to three weeks of the protocol which elicited the lowest $\mathrm{T}$ response $\left(\mathrm{T}_{\min }\right)$, and three weeks to the protocol which elicited the highest $\mathrm{T}$ response $\left(\mathrm{T}_{\max }\right)$ in a cross-over design. The protocols were the same as in the earlier investigation [25]. It was reported that body mass, 1RM bench press and leg press all showed significant increases after the period of $\mathrm{T}_{\max }$ training. When performing the $\mathrm{T}_{\min }$ period of training, $75 \%$ of athletes showed either no change or a significant decline in 1RM performance. The authors concluded by suggesting the ability of a resistance exercise protocol to induce an increase in free $\mathrm{T}$ is causatively linked to strength and bodyweight gains. The observation by Beaven et al. [25] that hormone response to certain resistance exercise protocols shows inter-individual variability has many practical implications. Athletes may be able to make further musculoskeletal gains by individually prescribed resistance exercise protocols based on acute $\mathrm{T}$ response.

Cortisol: $\mathrm{C}$ is a steroid hormone released by the adrenocortical glands under hypothalamic and pituitary control. C has catabolic effects on muscle tissue and has important metabolic functions such as influencing the metabolism of lipids, proteins and glucose [76]. It increases the mobilisation of fatty acids from fat reserves to active tissue and raises blood glucose [93]. Intense physical exercise causes an increase in $\mathrm{C}$ which may inhibit protein synthesis and consequently increases in muscle mass by its catabolic effect $[93,94]$. All protocols investigated by Beaven et al. [25] resulted in a decrease in C concentration with strength, hypertrophy, power and strength endurance protocols decreasing $C$ by $38.2 \pm 20.6 \%, 33.6 \pm 20.6 \%, 44.3 \pm 20.6 \%$ and 22.2 $\pm 20.6 \%$ respectively. These findings conflict with a number of studies who report acute increases in $\mathrm{C}$ in response to resistance exercise $[25,68,95]$. Beaven et al. [25] attributed these differences to the anticipatory anxious response to stressful events that cause an increase in $\mathrm{C}$ and suggested that by investigating individuals with experience of resistance training and using non-invasive saliva-obtained samples, the stress to individuals in the study was reduced. In exercising humans, $\mathrm{C}$ increases the availability of metabolic substrates for exercising musculature, protects from an over-reaction of the immune system to the stress of exercise and maintains normal vascular integrity [96]. C also prepares the body for the next bout of exercise explaining why postexercise levels of $\mathrm{C}$ are raised and the return to basal concentrations is prolonged. An important role of the acute $\mathrm{C}$ response is to meet the greater metabolic demand of resistance training $[97,98]$. In previous reports, acute $\mathrm{C}$ response has been highest when the overall stress of the training period has been very high and the response has been linked to the volume and/or intensity of total work $[68,91,95]$. Long-term resistance exercise may lead to an overall reduction of acute $\mathrm{C}$ response during exercise in men [32,91]. However, Cadore et al. [24] opposed this by suggesting that untrained and resistance trained men exhibited a similar $\mathrm{C}$ response to a resistance exercise stimulus. Nevertheless, an increased $\mathrm{T}$ response to resistance exercise in trained individuals was reported. These findings may suggest that the participants were in an increased state of anabolism due to an increase in protein synthesis rather than a reduction in protein degradation. However, this opposes the understanding that untrained individuals embarking on resistance training experience more drastic hypertrophy than trained individuals. This may be due to long-term resistance training attenuating the protein synthetic response to acute resistance exercise, by shortening the duration for which protein synthesis is elevated [59].

The ratio of $\mathrm{T} / \mathrm{C}$ is being intensively discussed and examined as a possible indicator of the anabolic/catabolic status of athletes. From a physiological view, the formation of such a quotient is problematic, as a number of other hormones also affect the anabolic/catabolic status of the body [99]. Addlercruetz et al. [100] however, suggested the T/C ratio as the most sensitive indicator of physical overload. Defining values of overload using this ratio have proven difficult due to high inter-individual variability [101]. Therefore, the ratio may be useful when investigating within-subjects effects however, caution should be used when applying this ratio to between subjects design. (Figure 3)

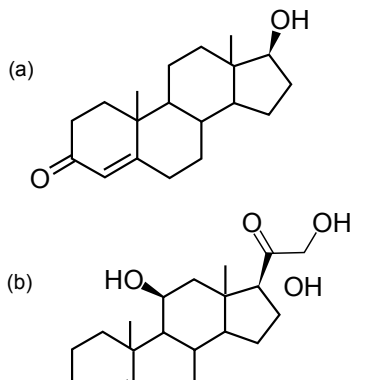

Figure 3: Line diagrams of steroid hormones (a) testosterone and (b) cortisol. 
IGF-1: IGF-1 is secreted as it is produced in the liver in response to GH-stimulated DNA synthesis [102] and remains the most prescribed signal for activating the signal transduction necessary for the initiation of protein translation after mechanical loading of the muscle [103]. IGF-1 is a potent activator of the Akt/mTOR signalling pathway with inhibition shown to prevent protein synthesis after RE [104, 105]. RE has been shown to increase concentrations of circulating and muscle IGF-1 although a number of studies have reported no change [106108]. The lack of change has been attributed to a delayed response of IGF-1 (3-9 h), following GH-stimulated mRNA synthesis with peak values not occurring until $16-28 \mathrm{~h}$ post-exercise [77,107]. RE alters concentrations of IGF binding proteins that influence the biological activity of IGF-1 $[108,109]$. IGF-1 stimulates muscle hypertrophy via phosphatidylinositol-3 kinase-(PI-3K) Akt-mTOR signalling. IGF-1 also increases the proliferation and differentiation of satellite cells to aid hypertrophy [110] and in response to mechanical overload, mechanogrowth factor (MGF; a splice variant of IGF-1) stimulates satellite cell activation [76]. IGF-1 is known to stimulate myoblast proliferation and differentiation in vitro as well as muscle protein synthesis [17,111]. Local expression of IGF-1 in skeletal muscle appears to be load dependent and acts independently of changes in serum GH or IGF-1 [112]. However, Wilborn et al. [113] reported increased mRNA expression of IGF-1 for protocols of $60-65 \% 1 \mathrm{RM} 4 \times 18-20$ repetitions and $80-85 \% 1 \mathrm{RM}$ $4 \times 8$-10 repetitions. No significant effect for RE protocol was reported for IGF-1, IGF-1 receptor or MGF suggesting that these isoforms may not be load dependent. Interestingly, volume was not controlled for by Wilborn et al. [113] and may have contributed to this indifference as the 60-65\% 1RM group experienced greater training volume. It would have been interesting if Wilborn et al. [113] had continued this study over a longer period to determine if hypertrophy, like muscle IGF-1, was equal for both protocols over time. Muscle IGF-1 is thought to induce myofiber hypertrophy by autocrine and/or paracrine action [111]. The effectiveness of muscle IGF-1 is dependent not only on its expression but also on its availability, which is controlled by a family of six IGF binding proteins and by the number of the IGF-1 receptor. As already suggested, expression of IGF-1 in muscle is independent of serum GH and IGF-1 and therefore, exogenous supplementation of serum GH or IGF-1 does not appear to stimulate muscular hypertrophy in the absence of applied load [114]. However, administration of IGF-1 directly into skeletal muscle does increase muscle mass suggesting that any increase in muscle IGF-1 availability may lead to hypertrophy [115]. Bamman et al. [108] hypothesised that muscle IGF-1 after a single bout of resistance exercise would be higher after eccentric contractions compared with concentric contractions. However, the results revealed that although MVC was decreased for longer and muscle soreness was perceived higher after eccentric exercise, serum IGF-1 expression showed no significant difference between the muscle actions. Nevertheless, local IGF-1 is independent of serum IGF-1 and therefore IGF-binding protein-4 (known to inhibit IGF-1) mRNA was reduced significantly after eccentric loading which lead to an increase in skeletal IGF1 mRNA. This would lead to increased IGF-1 availability within the muscle after mechanical loading. Therefore, the authors suggested that IGF-1 was linked to mechanisms involved in tissue regeneration caused by muscle damage. This muscle damage may not lead to hypertrophy however as downhill running and other endurance exercises with large eccentric component cause significant muscle damage, yet endurance exercise is not a potent hypertrophic stimulus [91].

Insulin: Insulin causes cells in the liver, muscle, and adipose tissue to uptake glucose from the blood, storing it as glycogen in the liver and muscle, and stopping use of fat as an energy source. During exercise, insulin secretion is reduced to allow more glucose to the muscle cells.
However, it is the aim during RE to minimise this reduction in insulin as it has been shown to have significant effect on muscle protein synthesis when adequate amino acid concentrations are available, by reducing protein catabolism [115]. Exercise-induced mRNA transcription and synthesis of proteins is only fully activated when plasma insulin levels are elevated [116]. Elevations in insulin are expected to inhibit AMPK activity, therefore promoting protein synthesis. Insulin likely exerts its effect on translation through the Akt-mTOR signal transduction pathway [117]. Serum insulin concentrations parallel changes in blood glucose, and the response is enhanced when protein and carbohydrates are ingestion prior to, during, or after a workout [107,115]. A potent anabolic hormone in its normal physiological range; insulin is mostly affected by blood glucose concentrations and dietary intake. Ingestion of carbohydrates, amino acids or a combination of both prior to, during, or after a workout is recommended for maximising insulin's effects on tissue anabolism [91]. Supplementation before or during RE may maximize benefits as a consequence of increased muscular blood flow and subsequent amino acid delivery.

GH: The acidophilic cells of the anterior pituitary secrete molecules that make up the family of $\mathrm{GH}$ polypeptides. The most commonly studied GH isoform, the $22 \mathrm{kD}$ molecule, consists of 191 amino acids [118]. Other isoforms also exist and appear to function similarly to the $22 \mathrm{kD}$ molecule in promoting tissue anabolism. RE has been shown to acutely elevate many of the GH variants [119]. GH binding to its receptor initiates janus kinase 2 (JAK2) signalling. JAK2 signaling activates PI$3 \mathrm{~K}$. As PI-3K is proximal to Akt-mTOR signalling, it appears that REinduced elevations in GH promote translational efficiency and muscle anabolism [13]. Animal studies support this, as GH injection into pigs resulted in enhance translational efficiency and protein synthesis $[120,121]$. This RE-induced elevation in GH has been shown through 30 min post-exercise [77]. The magnitude of this response appears dependent upon the amount of muscle mass recruited contraction velocity volume intensity and rest intervals [3,21,67,68,80,95]. As with $\mathrm{T}$, greater $\mathrm{GH}$ acute responses have been reported with workouts consisting of multiple-sets [68]. GH appears to be highly influenced by the volume of the RE protocol. GH response appears to be most substantial when a protocol elicits a high blood lactate concentration [67]. To evoke a substantial blood lactate response, a RE protocol should include moderate to high load, high volume, short rest intervals and activate large muscle groups. High correlations between serum GH and blood lactate have been reported and Kraemer et al. [77] proposed the accumulation of $\mathrm{H}^{+}$produced by lactic acid acidosis as the primary factor influencing GH release [95]. Mulligan et al. [122] supported this hypothesis by suggesting that GH response was attenuated following induced alkalosis during high-intensity cycling. Although endurance exercise may transiently increases many of the anabolic hormones, prolonged endurance exercise is not a potent stimulus of hypertrophy as it does not recruit enough muscle fibres (androgen receptors are only activated if the motor unit is activated), specifically type II fibres (known to be most susceptible to hypertrophy) or stimulate protein synthesis via the Akt-mTOR pathway. Breath-holding, Hypoxia, protein catabolism and acid base shifts have also been reported to influence GH release [77]. Therefore, resistance exercise is a potent stimulus for increasing GH as long as exercise intensity and volume is sufficient [123]. A number of studies have reported a correlation between the total work of a RE protocol and the acute GH response [95,122]. Mostly, these studies compare typical strength protocols (high load, low repetitions, and long rest) with typical hypertrophy protocols (moderate load, moderate repetitions, short rest) or a typical strength endurance protocol (low load, high repetitions and short rest). Commonly the strength endurance protocol results in the greatest 


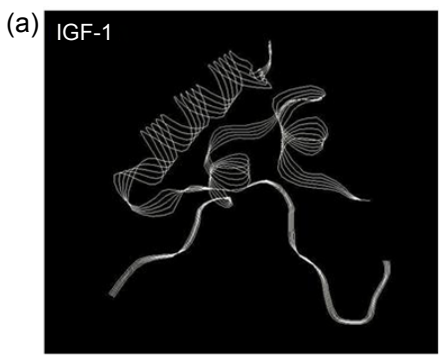

(b) Human insulin
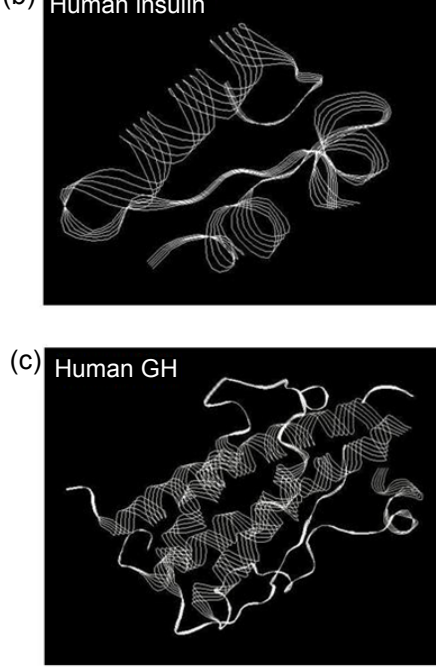

Figure 4: RASMOL generated structures of protein hormones using RCSB Protein Data Bank (PDB) files (http://www.pdb.org/pdb/home/home.do):

(a) Insulin-like growth factor-1 (1 polypeptide chain of 70 residues) PDB reference $1 \mathrm{GZR}$.

(b) Human insulin (2 polypeptide chains A 21 residues \& B 30 residues) PDB reference $3 \mid 3 Z$.

(c) Human growth hormone (1 polypeptide chain of 191 amino acid residues,) PDB reference $1 \mathrm{HGU}$.

acute elevation in GH. However, Campos et al. [28] demonstrated that this type of protocol does not result in increased muscular CSA and was the least effective at increasing strength compared to a strength and hypertrophy protocol. These findings suggest that although GH may influence the muscular adaptations to RE, the extent to which GH is acutely elevated is not the ultimate factor determining adaptations. Interestingly, Goto et al. [65] reported that the addition of a single set of high repetitions with 50\% 1RM to the end of a strength regimen elicited a much higher $\mathrm{GH}$ response than with the strength protocol alone. However, the hypertrophy protocol utilised elicited a higher acute $\mathrm{GH}$ response than the strength protocol alone and the strength protocol with the addition of a high-repetitions set. However, the combination of the strength protocol and the high-repetition set did result in increased CSA when compared to the strength protocol without the addition of the high-repetition set (Figure 4).

\section{Conclusion}

Traditionally, 1-6RM, 8-12RM and 15+RM loads are recommended to maximize strength, hypertrophy and local muscular endurance, respectively with light loads ( $40 \% 1 \mathrm{RM})$ performed explosively recommended for power training. Most research concerning RE conforms to these ideals however, athletes may experience enhanced adaptations when performing a RE protocol that maximizes hormonal response as this varies inter-individually for the same protocols. To maximize hypertrophy, load needs to be substantial enough to recruit fast twitch muscle fibres but light enough to enable short rest periods and a volume capable of causing metabolic stress. This type of protocol maximizes hormonal response. Approximately 5-6RM appears optimal for increasing maximal dynamic strength. Much smaller loads have increased strength in untrained participants but as individuals become more experienced in $\mathrm{RE}$, a greater load is required to stimulate overload. Commonly, compound exercises (Olympic lifts, dead lifts, squats, bench press) have been prescribed to maximize hypertrophy as they cause a high metabolic stress and stimulate a large hormonal response, thereby increasing protein synthesis. However, hypertrophy has been reported in smaller muscle groups without an anabolic hormone response to the protocol. This suggests that a combination of central and peripheral factors stimulate hypertrophy. Compound exercises are advised for athletes as in the majority of events, large muscle groups are the most utilised (often through locomotion) however, RE should be tailored to the individual athlete's needs. A combination of eccentric and concentric muscle actions is advised for strength and hypertrophy training. Muscle adaptations appear specific to training and isotonic muscle actions are common in most weight lifting events. The exclusion of eccentric muscle actions in a RE protocol has been shown to attenuate muscle growth.

Superior results have been reported for all protocols when incorporating periodization into a training program. A dailyundulating-periodization; characterised by cycling training loads over a short period of time (E.g. 6RM Monday, 10RM Wednesday, 14RM Friday) appears superior to linear periodization and non-periodized training possibly due to more regular overload.

Availability of amino acids is important for protein synthesis associated with hypertrophy and inclusion of $\mathrm{CHO}$ may maximize the effect of insulin with creatine also increasing lean body mass. Therefore, a combination of $\mathrm{CHO}$, protein and creatine would be most beneficial to consume pre-, during-, and post-exercise to maximize hypertrophy and recovery. However, creatine is not recommended for athletes who compete in events that require acceleration of one's own body mass. A number of other factors affect the adaptations to RE including age, genetics, gender, time of day trained at, posture and living habits but these are outside the scope of this review.

With regards to previous and future research, it is clear that acute program variables are difficult to examine in isolation and this issue proves problematic in RE research. Therefore, it is difficult to make any certain conclusions about acute RE variables as altering one will normally impact on another. The issue of hypertrophy in the absence of elevated anabolic hormones is an interesting phenomenon that deserves further investigation. Although this phenomenon has been observed, hypertrophy programs have previously been prescribed based on anabolic hormone response and the findings of Cadore et al. [24] suggest that a hypertrophy is superior in increasing muscle growth compared to a strength or muscular endurance protocol. Therefore, another mechanism other than hormone response may determine why a program utilising repetitions of approximately 10 is superior in eliciting hypertrophy. Future investigations could aim to discover this mechanism. With regards to acute program variables, it is still unclear whether a dose response exists; i.e. would utilising 5, 4, 3, 2 and, 1 sets of an exercise result in a stepwise increase in strength or hypertrophy. Then, could this be extrapolated as far as 10 sets and beyond. This hole in the literature is apparent for all program variables, however may be most applicable to volume. 


\section{References}

1. Kraemer WJ (1983) Exercise Prescription in Weight Training: Manipulating Program Variables. Nat Strength Cond Assoc J 5: 58-59.

2. Chilibeck PD, Calder AW, Sale DG, Webber CE (1998)A comparison of strength and muscle mass increases during resistance training in young women. Eur J Appl Physiol Occup Physiol 77: 170-175

3. Stone MH, Plisk SS, Stone ME, Schilling BK, O'Bryant HS, et al. (1998) Athletic performance development: Volume load - 1 set vs. Multiple sets, training velocity and training variation. Strength Cond J 20: 22-31.

4. Wilkinson SB, Tarnopolsky MA, Grant EJ, Correia CE, Phillips SM (2006) Hypertrophy with unilateral resistance exercise occurs without increases in endogenous anabolic hormone concentration. Eur J Appl Physiol 98: 546-555.

5. West DWD, Burd NA, Tang JE, Moore DR, Staples AW, et al. (2010) Elevations in ostensibly anabolic hormones with resistance exercise enhance neither training-induced muscle hypertrophy nor strength of the elbow flexors. J App Physiol 108: 60-67.

6. Welle S, Totterman S, Thornton C (1996) Effect of age on muscle hypertrophy induced by resistance training. J Gerontol A Biol Sci Med Sci 51: M270-275.

7. Abe T, DeHoyos DV, Pollock ML, Garzarella L (2000) Time course for strength and muscle thickness changes following upper and lower body resistance training in men and women. Eur J Appl Physiol 81: 174-180.

8. Cureton KJ, Collins MA, Hill DW, McElhannon FM Jr (1988) Muscle hypertrophy in men and women. Med Sci Sports Exerc 20: 338-344.

9. Kadi F, Bonnerud P, Eriksson A, Thornell LE (2000) The expression of androgen receptors in human neck and limb muscles: effects of training and self-administration of androgenic-anabolic steroids. Histochem Cell Biol 113 25-29.

10. Sakamoto A, Sinclair PJ (2006) Effect of movement velocity on the relationship between training load and the number of repetitions of bench press. J Strength Cond Res 20: 523-527.

11. Coburn JW, Housh TJ, Malek MH, Weir JP, Cramer JT, et al. (2006) Neuromuscular responses to three days of velocity-specific isokinetic training J Strength Cond Res 20: 892-898.

12. Kanehisa $\mathrm{H}$, Miyashita $\mathrm{M}$ (1983) Specificity of velocity in strength training. Eur J Appl Physiol Occup Physiol 52: 104-106.

13. Spiering BA, Kraemer WJ, Anderson JM, Armstrong LE, Nindl BC, et al. (2008) Resistance exercise biology: Manipulation of resistance exercise programme variables determines the responses of cellular and molecular signaling pathways. Sports Med 38: 527-540.

14. Eliasson J, Elfegoun T, Nilsson J, Köhnke R, Ekblom B, et al. (2006) Maxima lengthening contractions increase p70 S6 kinase phosphorylation in human skeletal muscle in the absence of nutritional supply. Am J Physiol Endocrino Metab 291: E1197-1205.

15. Long YC, Widegren U, Zierath JR (2004) Exercise-induced mitogen-activated protein kinase signalling in skeletal muscle. Proc Nutr Soc 63: 227-232.

16. Garma T, Kobayashi C, Haddad F, Adams GR, Bodell PW, et al. (2007) Similar acute molecular responses to equivalent volumes of isometric, lengthening, or shortening mode resistance exercise. J Appl Physiol 102: 135-143.

17. Hather BM, Tesch PA, Buchanan P, Dudley GA (1991) Influence of eccentric actions on skeletal muscle adaptations to resistance training. Acta Physio Scand 143: 177-185.

18. Spreuwenberg LP, Kraemer WJ, Spiering BA, Volek JS, Hatfield DL, et al. (2006) Influence of exercise order in a resistance-training exercise session. J Strength Cond Res 20: 141-144.

19. Dias I, de Salles BF, Novaes J, Costa PB, Simão R (2010) Influence of exercise order on maximum strength in untrained young men. J Sci Med Sport 13: 65-69.

20. Augustsson J, Thomeé R, Hörnstedt P, Lindblom J, Karlsson J, et al. (2003) Effect of pre-exhaustion exercise on lower-extremity muscle activation during a leg press exercise. J Strength Cond Res 17: 411-416.

21. Baker D, Newton RU (2005) Acute effect on power output of alternating an agonist and antagonist muscle exercise during complex training. J Strength Cond Res 19: 202-205.

22. American College of Sports Medicine (2009) American College of Sports Medicine position stand. Progression models in resistance training for healthy adults. Med Sci Sports Exerc 41: 687-708.
23. Ratamess NA, Falvo MJ, Mangine GT, Hoffman JR, Faigenbaum AD, et al (2007) The effect of rest interval length on metabolic responses to the bench press exercise. Eur J Appl Physiol 100: 1-17.

24. Lusa Cadore E, Lhullier FL, Arias Brentano M, Marczwski Da Silva E, Bueno Ambrosini M, et al. (2009) Salivary hormonal responses to resistance exercise in trained and untrained middle-aged men. J Sports Med Phys Fitness 49: 301 307.

25. Beaven CM, Gill ND, Cook CJ (2008) Salivary testosterone and cortiso responses in professional rugby players after four resistance exercise protocols. J Strength Cond Res 22: 426-432.

26. Hakkinen K, Alen M, Komi PV (1985) Changes in isometric force-time and relaxation-time, electromyographic and muscle-fiber characteristics of human skeletal-muscle during strength training and detraining. Acta Physiol Scand 125: $573-585$

27. Weiss LW, Coney HD, Clark FC (1999) Differential functional adaptations to short-term low-, moderate-, and high-repetition weight training. J Strength Cond Res 13: 236-241.

28. Campos GE, Luecke TJ, Wendeln HK, Toma K, Hagerman FC, et al. (2002) Muscular adaptations in response to three different resistance-training regimens: specificity of repetition maximum training zones. Eur J Appl Physio 88: $50-60$.

29. Berger RA (1962) Optimum repetitions for the development of strength. Res Q 33: 334-338.

30. O'Shea P (1966) Effects of selected weight training programs on the development of strength and muscle hypertrophy. Res $Q 37$ : 95-102.

31. Wilson GJ, Newton RU, Murphy AJ, Humphries BJ (1993) The optimal training load for the development of dynamic athletic performance. Med Sci Sports Exerc 25: 1279-1286.

32. Staron RS, Karapondo DL, Kraemer WJ, Fry AC, Gordon SE, et al. (1994) Skeletal muscle adaptations during early phase of heavy-resistance training in men and women. J Appl Physiol 76: 1247-1255.

33. Kraemer WJ (1997) A series of studies - The physiological basis for strength training in American football: Fact over philosophy. J Strength Cond ReS 11 131-142.

34. Nader GA, Esser KA (2001) Intracellular signaling specificity in skeletal muscle in response to different modes of exercise. J Appl Physiol 90: 1936-1942.

35. Atherton PJ, Babraj J, Smith K, Singh J, Rennie MJ, et al. (2005) Selective activation of AMPK-PGC-1alpha or PKB-TSC2-mTOR signaling can explain specific adaptive responses to endurance or resistance training-like electrical muscle stimulation. FASEB J 19: 786-788.

36. Hornberger TA, Chien S (2006) Mechanical stimuli and nutrients regulate rapamycin-sensitive signaling through distinct mechanisms in skeletal muscle. J Cell Biochem 97: 1207-1216.

37. Bodine SC, Stitt TN, Gonzalez M, Kline WO, Stover GL, et al. (2001) Akt/mTOR pathway is a crucial regulator of skeletal muscle hypertrophy and can prevent muscle atrophy in vivo. Nat Cell Biol 3: 1014-1019.

38. Terada N, Patel HR, Takase K, Kohno K, Nairn AC, et al. (1994) Rapamycin selectively inhibits translation of mRNAs encoding elongation factors and ribosomal proteins. Proc Natl Acad Sci U S A 91: 11477-11481.

39. Henneman E, Somjen G, Carpenter DO (1965) Functional Significance of Cell Size in Spinal Motoneurons.

40. McCaulley GO, McBride JM, Cormie P, Hudson MB, Nuzzo JL, et al. (2009) Acute hormonal and neuromuscular responses to hypertrophy, strength and power type resistance exercise. Eur J Appl Physiol 105: 695-704.

41. Linnamo V, Newton RU, Häkkinen K, Komi PV, Davie A, et al. (2000) Neuromuscular responses to explosive and heavy resistance loading. J Electromyogr Kinesiol 10: 417-424.

42. Pincivero DM, Gandhi V, Timmons MK, Coelho AJ (2006) Quadriceps femoris electromyogram during concentric, isometric and eccentric phases of fatiguing dynamic knee extensions. J Biomech 39: 246-254

43. McCall GE, Byrnes WC, Dickinson A, Pattany PM, Fleck SJ (1996) Muscle fiber hypertrophy, hyperplasia, and capillary density in college men after resistance training. J Appl Physiol 81: 2004-2012.

44. Trappe SW, Trappe TA, Lee GA, Widrick JJ, Costill DL, et al. (2001) Comparison of a space shuttle flight (STS-78) and bed rest on human muscle function. 
Appl Physiol 91: 57-64.

45. Kraemer WJ, Ratamess NA (2004) Fundamentals of resistance training: progression and exercise prescription. Med Sci Sports Exerc 36: 674-688.

46. Peterson MD, Rhea MR, Alvar BA (2005) Applications of the dose-response for muscular strength development: a review of meta-analytic efficacy and reliability for designing training prescription. J Strength Cond Res 19: 950-958.

47. Prestes J, Frollini AB, de Lima C, Donatto FF, Foschini D, et al. (2009) Comparison between linear and daily undulating periodized resistance training to increase strength. J Strength Cond Res 23: 2437-2442.

48. Rhea MR, Ball SD, Phillips WT, Burkett LN (2002) A comparison of linear and daily undulating periodized programs with equated volume and intensity for strength. J Strength Cond Res 16: 250-255.

49. Selye H (1974) Stress without distress. New York: JB Lippincott Company

50. Tran QT, Docherty D, Behm D (2006) The effects of varying time under tension and volume load on acute neuromuscular responses. Eur J Appl Physiol 98: $402-410$

51. Borst SE, De Hoyos DV, Garzarella L, Vincent K, Pollock BH, et al. (2001) Effects of resistance training on insulin-like growth factor-I and IGF binding proteins. Med Sci Sports Exerc 33: 648-653.

52. Willoughby DS, Chilek DR, Schiller DA, Coast JR (1991) The metabolic effects of 3 different free weight parallel squatting intensities. J Hum Movement Stud 21: 53-67.

53. Dudley GA, Djamil R (1985) Incompatibility of endurance- and strength-training modes of exercise. J Appl Physiol 59: 1446-1451.

54. Hortobágyi T, Barrier J, Beard D, Braspennincx J, Koens P, et al. (1996) Greater initial adaptations to submaximal muscle lengthening than maximal shortening J Appl Physiol 81: 1677-1682.

55. Housh DJ, Housh TJ, Johnson GO, Chu WK (1992) Hypertrophic response to unilateral concentric isokinetic resistance training. J Appl Physiol 73: 65-70.

56. Ostrowski KJ, Wilson GJ, Weatherby R, Murphy PW, Lyttle AD (1997) The effect of weight training volume on hormonal output and muscular size and function. J Strength Cond Res 11: 148-154.

57. Capen EK (1956) Study of four programs of heavy resistance exercises for development of muscular strength. Res Q 27: 132-142.

58. Krieger JW (2009) Single versus multiple sets of resistance exercise: a metaregression. J Strength Cond Res 23: 1890-1901.

59. Tang JE, Perco JG, Moore DR, Wilkinson SB, Phillips SM (2008) Resistance training alters the response of fed state mixed muscle protein synthesis in young men. Am J Physiol Regul Integr Comp Physiol 294: R172-178.

60. MacDougall JD, Gibala MJ, Tarnopolsky MA, MacDonald JR, Interisano SA et al. (1995) The time course for elevated muscle protein synthesis following heavy resistance exercise. Can J Appl Physiol 20: 480-486.

61. Phillips SM (2000) Short-term training: When do repeated bouts of resistance exercise become training? Can J Appl Physiol 25: 185-193.

62. Rhea MR, Alvar BA, Ball SD, Burkett LN (2002) Three sets of weight training superior to 1 set with equal intensity for eliciting strength. J Strength Cond Res 16: $525-529$.

63. Moss BM, Refsnes PE, Abildgaard A, Nicolaysen K, Jensen J (1997) Effects of maximal effort strength training with different loads on dynamic strength, crosssectional area, load-power and load-velocity relationships. Eur J Appl Physio Occup Physiol 75: 193-199.

64. Marx JO, Ratamess NA, Nindl BC, Gotshalk LA, Volek JS, et al. (2001) Lowvolume circuit versus high-volume periodized resistance training in women. Med Sci Sports Exerc 33: 635-643.

65. Goto K, Nagasawa M, Yanagisawa O, Kizuka T, Ishii N, et al. (2004) Muscular adaptations to combinations of high- and low-intensity resistance exercises. $J$ Strength Cond Res 18: 730-737

66. Creer A, Gallagher P, Slivka D, Jemiolo B, Fink W, et al. (2005) Influence of muscle glycogen availability on ERK1/2 and Akt signaling after resistance exercise in human skeletal muscle. J Appl Physiol 99: 950-956.

67. Kraemer WJ, Gordon SE, Fleck SJ, Marchitelli LJ, Mello R, et al. (1991) Endogenous anabolic hormonal and growth factor responses to heavy resistance exercise in males and females. Int J Sports Med 12: 228-235.
68. Gotshalk LA, Loebel CC, Nindl BC, Putukian M, Sebastianelli WJ, et al. (1997) Hormonal responses of multiset versus single-set heavy-resistance exercise protocols. Can J Appl Physiol 22: 244-255

69. Kraemer WJ, Noble BJ, Clark MJ, Culver BW (1987) Physiologic responses to heavy-resistance exercise with very short rest periods. Int J Sports Med 8 : 247-252.

70. Behm DG, Reardon G, Fitzgerald J, Drinkwater E (2002) The effect of 5, 10, and 20 repetition maximums on the recovery of voluntary and evoked contractile properties. J Strength Cond Res 16: 209-218.

71. Miranda H, Simao R, Moreira LM, de Souza RA, se Souza JAA, et al (2009) Effect of rest interval length on the volume completed during upper body resistance exercise. J Sport Sci Med 8: 388-392.

72. Robinson JM, Stone MH, Johnson RL, Penland CM, Warren BJ, et al. (1995) Effects of Different Weight Training Exercise/Rest Intervals on Strength, Power, and High Intensity Exercise Endurance. J Strength Cond Res 9: 216-221.

73. Ahtiainen JP, Pakarinen A, Alen M, Kraemer WJ, Häkkinen K (2005) Short vs long rest period between the sets in hypertrophic resistance training: influence on muscle strength, size, and hormonal adaptations in trained men. J Strength Cond Res 19: 572-582.

74. Crewther BT, Lowe T, Weatherby RP, Gill N, Keogh J (2009) Neuromuscular performance of elite rugby union players and relationships with salivary hormones. J Strength Cond Res 23: 2046-2053.

75. Vermeulen A, Verdonck L, Kaufman JM (1999) A critical evaluation of simple methods for the estimation of free testosterone in serum. J Clin Endocrino Metab 84: 3666-3672.

76. Florini JR (1987) Hormonal control of muscle growth. Muscle Nerve 10: 577598

77. Kraemer WJ, Fleck SJ, Dziados JE, Harman EA, Marchitelli LJ, et al. (1993) Changes in hormonal concentrations after different heavy-resistance exercise protocols in women. J Appl Physiol 75: 594-604.

78. Kvorning T, Andersen M, Brixen K, Madsen K (2006) Suppression of endogenous testosterone production attenuates the response to strength training: a randomized, placebo-controlled, and blinded intervention study. Am J Physiol Endoc Metab 291: 1325-1332.

79. Hansen S, Kvorning T, Kjaer M, Sjøgaard G (2001) The effect of short-term strength training on human skeletal muscle: the importance of physiologically elevated hormone levels. Scand J Med Sci Sports 11: 347-354.

80. Ahtiainen JP, Pakarinen A, Kraemer WJ, Häkkinen K (2003) Acute hormona and neuromuscular responses and recovery to forced vs maximum repetitions multiple resistance exercises. Int J Sports Med 24: 410-418.

81. Phillips SM, Tipton KD, Aarsland AA, Wolf SE, Wolfe RR (1997) Time course of mixed muscle protein synthesis following resistance exercise in humans. Med Sci Sports Exerc 29: 230.

82. Sinha-Hikim I, Artaza J, Woodhouse L, Gonzalez-Cadavid N, Singh AB, et al. (2002) Testosterone-induced increase in muscle size in healthy young men is associated with muscle fiber hypertrophy. Am J Physiol Endocrinol Metab 283: E154-164.

83. Zitzmann M, Nieschlag E (2003) Effects of androgen replacement on metabolism and physical performances in male hypogonadism. J Endocrino Invest 26: 886-892.

84. Mauras N, Hayes V, Welch S, Rini A, Helgeson K, et al. (1998) Testosterone deficiency in young men: marked alterations in whole body protein kinetics, strength, and adiposity. J Clin Endocrinol Metab 83: 1886-1892.

85. Beaven CM, Cook CJ, Gill ND (2008) Significant strength gains observed in rugby players after specific resistance exercise protocols based on individual salivary testosterone responses. J Strength Cond Res 22: 419-425.

86. West DW, Kujbida GW, Moore DR, Atherton P, Burd NA, et al. (2009) Resistance exercise-induced increases in putative anabolic hormones do not enhance muscle protein synthesis or intracellular signalling in young men. $J$ Physiol 587: 5239-5247.

87. McCall GE, Byrnes WC, Fleck SJ, Dickinson A, Kraemer WJ (1999) Acute and chronic hormonal responses to resistance training designed to promote muscle hypertrophy. Can J Appl Physiol 24: 96-107

88. Inoue K, Yamasaki S, Fushiki T, Okada Y, Sugimoto E (1994) Androgen recepto antagonist suppresses exercise-induced hypertrophy of skeletal muscle. Eur $J$ Appl Physiol Occup Physiol 69: 88-91. 
Citation: Hayes LD, Bickerstaff GF, Baker JS (2013) Acute Resistance Exercise Program Variables and Subsequent Hormonal Response. J Sports Med Doping Stud 3: 125. doi:10.4172/2161-0673.1000125

Page 10 of 10

89. Lee WJ, McClung J, Hand GA, Carson JA (2003) Overload-induced androgen receptor expression in the aged rat hindlimb receiving nandrolone decanoate. J Appl Physiol 94: 1153-1161.

90. Kraemer WJ, Hatfield DL, Volek JS, Fragala MS, Vingren JL, et al. (2009) Effects of amino acids supplement on physiological adaptations to resistance training. Med Sci Sports Exerc 41: 1111-1121.

91. Kraemer WJ, Patton JF, Gordon SE, Harman EA, Deschenes MR, et al. (1995) Compatibility of high-intensity strength and endurance training on hormonal and skeletal muscle adaptations. J Appl Physiol 78: 976-989.

92. Kraemer WJ, Loebel CC, Volek JS, Ratamess NA, Newton RU, et al. (2001) The effect of heavy resistance exercise on the circadian rhythm of salivary testosterone in men. Eur J Appl Physiol 84: 13-18.

93. Vale RG, de Oliveira RD, Pernambuco CS, de Meneses YP, Novaes Jda S, et al. (2009) Effects of muscle strength and aerobic training on basal serum levels of IGF-1 and cortisol in elderly women. Arch Gerontol Geriatr 49: 343-347.

94. Timon R, Olcina G, Tomas-Carus P, Muñoz D, Toribio F, et al. (2009) Urinary steroid profile after the completion of concentric and concentric/eccentric trials with the same total workload. J Physiol Biochem 65: 105-112.

95. Smilios I, Pilianidis T, Karamouzis M, Tokmakidis SP (2003) Hormonal responses after various resistance exercise protocols. Med Sci Sports Exerc 35: $644-654$

96. Häkkinen K, Pakarinen A (1993) Acute hormonal responses to two different fatiguing heavy-resistance protocols in male athletes. J Appl Physiol 74: 882887.

97. Duclos M (2008) A critical assessment of hormonal methods used in monitoring training status in athletes. Int Sportmed J 9: 56-66.

98. Duclos M, Gouarne C, Bonnemaison D (2003) Acute and chronic effects of exercise on tissue sensitivity to glucocorticoids. J Appl Physiol 94: 869-875.

99. Pfeffer LA, Brisson BK, Lei H, Barton ER (2009) The insulin-like growth factor (IGF)-I E-peptides modulate cell entry of the mature IGF-I protein. Mol Biol Cell 20: $3810-3817$

100. Adlercreutz $\mathrm{H}$, Härkönen $\mathrm{M}$, Kuoppasalmi K Näveri $\mathrm{H}$, Huhtaniemi I, et al. (1986) Effect of training on plasma anabolic and catabolic steroid hormones and their response during physical exercise. Int J Sports Med 7 Suppl 1: 27-28.

101. Flynn MG, Pizza FX, Boone JB Jr, Andres FF, Michaud TA, et al. (1994) Indices of training stress during competitive running and swimming seasons. Int J Sports Med 15: 21-26.

102. Fry AC, Kraemer WJ, Ramsey LT (1998) Pituitary-adrenal-gonadal responses to high-intensity resistance exercise overtraining. J Appl Physiol 85: 23522359.

103. Adams GR (2002) Invited Review: Autocrine/paracrine IGF-I and skeletal muscle adaptation. J Appl Physiol 93: 1159-1167.

104. Glass DJ (2005) Skeletal muscle hypertrophy and atrophy signaling pathways. Int J Biochem Cell Biol 37: 1974-1984.

105.Barton ER (2006) Viral expression of insulin-like growth factor-I isoforms promotes different responses in skeletal muscle. J Appl Physiol 100: 17781784 .

106. Fedele MJ, Lang CH, Farrell PA (2001) Immunization against IGF-I prevents increases in protein synthesis in diabetic rats after resistance exercise. Am J Physiol Endocrinol Metab 280: E877-885.

107. Chandler RM, Byrne HK, Patterson JG, Ivy JL (1994) Dietary supplements affect the anabolic hormones after weight-training exercise. J Appl Physiol 76: 839-845.
08. Bamman MM, Shipp JR, Jiang J, Gower BA, Hunter GR, et al. (2001) Mechanical load increases muscle IGF-I and androgen receptor mRNA concentrations in humans. Am J Physiol Endocrinol Metab 280: E383-390.

109. Nindl BC, Kraemer WJ, Hymer WC (2000) Immunofunctional vs immunoreactive growth hormone responses after resistance exercise in men and women. Growth Horm IGF Res 10: 99-103.

110. Hawke TJ (2005) Muscle stem cells and exercise training. Exerc Sport Sci Rev 33: 63-68.

111. Florini JR, Ewton DZ, Coolican SA (1996) Growth hormone and the insulin-like growth factor system in myogenesis. Endocr Rev 17: 481-517.

112. Jurasinski CV, Vary TC (1995) Insulin-like growth factor I accelerates protein synthesis in skeletal muscle during sepsis. Am J Physiol 269: E977-981.

113. Wilborn CD, Taylor LW, Greenwood M, Kreider RB, Willoughby DS (2009) Effects of different intensities of resistance exercise on regulators of myogenesis. J Strength Cond Res 23: 2179-2187.

114. Allen DL, Linderman JK, Roy RR, Grindeland RE, Mukku V, et al. (1997) Growth hormone/IGF-I and/or resistive exercise maintains myonuclear number in hindlimb unweighted muscles. J Appl Physiol 83: 1857-1861.

115. Adams GR, McCue SA (1998) Localized infusion of IGF-I results in skeletal muscle hypertrophy in rats. J Appl Physiol 84: 1716-1722.

116. Wolfe RR (2000) Effects of insulin on muscle tissue. Curr Opin Clin Nutr Metab Care 3: 67-71.

117. Kuo CH, Hunt DG, Ding Z, Ivy JL (1999) Effect of carbohydrate supplementation on postexercise GLUT-4 protein expression in skeletal muscle. J Appl Physiol 87: 2290-2295.

118. Kimball SR, Farrell PA, Jefferson LS (2002) Invited Review: Role of insulin in translational control of protein synthesis in skeletal muscle by amino acids or exercise. J Appl Physiol 93: 1168-1180.

119. Kraemer WJ, Clemson A, Triplett NT, Bush JA, Newton RU, et al. (1996) The effects of plasma cortisol elevation on total and differential leukocyte counts in response to heavy-resistance exercise. Eur J Appl Physiol Occup Physio 73: 93-97.

120. Nindl BC, Kraemer WJ, Marx JO, Arciero PJ, Dohi K, et al. (2001) Overnigh responses of the circulating IGF-I system after acute, heavy-resistance exercise. J Appl Physiol 90: 1319-1326.

121. Bush JA, Kimball SR, O'Connor PM, Suryawan A, Orellana RA, et al. (2003) Translational control of protein synthesis in muscle and liver of growth hormone-treated pigs. Endocrinology 144: 1273-1283.

122. Mulligan SE, Fleck SJ, Gordon SE, Koziris LP, Triplett-McBride NT, et al (1996) Influence of Resistance Exercise Volume on Serum Growth Hormone and Cortisol Concentrations in Women. J Strength Cond Res 10: 256-262.

123. Hoffman JR, Im J, Rundell KW, Kang J, Nioka S, et al. (2003) Effect of muscle oxygenation during resistance exercise on anabolic hormone response. Med Sci Sports Exerc 35: 1929-1934. 